\title{
Particle Sizing and Spray Analysis
}

\author{
Norman Chigier \\ Carnegie-Mellon University \\ Department of Mechanical Engineering \\ Pittsburgh, Pennsylvania 15213
}

\author{
Gerald Stewart \\ Aerodyne Research, Inc. \\ 45 Manning Road \\ Billerica, Massachusetts 01821
}

The measurement of particle size and velocity in particle laden flows is a subject of interest in a variety of industrial applications. In combustion systems for electricity generation, industriai processes and heating, and transportation, where liquid and solid fuels are injected into air streams for burning in furnaces, boilers, and gas turbine and diesel engines, the initial size and velocity distributions of particles are determining factors in the overall combustion efficiency and the emission of pollutants and particulates. In the design of injectors and burners for the atomization of liquid fuels, a great deal of attention is being focused on developing instrumentation for the accurate measurement of size and velocity distributions in sprays as a function of space and time. Most recent advances in optical engineering techniques using lasers for particle measurement have focused on detailed spray characterization, where there is a major concern with spherical liquid droplets within the size range of 1 to $500 \mu \mathrm{m}$ in diameter, with droplet velocities within the range of 1 to $100 \mathrm{~m} / \mathrm{s}$, and the requirement for making in situ measurements of moving particles by nonintrusive optical probes. The instruments being developed for spray analysis have much wider applications. These include measurement in particle laden flows encountered in a variety of industrial processes with solid particles in gas and liquid streams and liquid particles in gas streams. Sprays used in agriculture, drying, food processing, coating of materials, chemical processing, clean rooms, pharmaceuticals, plasma spraying, and icing wind tunnels are examples of systems for which information is being sought on particle and fluid dynamic interactions in which there is heat, mass, and momentum transfer in turbulent reacting flows.

Even though there has been a general interest in these types of measurements for many years, the rapid developments in laser, electronic, computing, instrumentation, and control technologies in recent years have resulted in much greater activity in research, development, and commercialization of a variety of instruments. As a result of a demand and a market for instruments covering a wide spectrum of price and degree of sophistication, novel methods are being devised and refinements and simplifications are being introduced in order to develop instruments that are accurate, reliable, and rugged for use in field, industrial, and atmospheric environments.

The laser optical particle sizing techniques discussed in this special issue of Optical Engineering are subdivided into three categories: (1) imaging-microphotography, holography, cinematography; (2) single particle counting; and (3) ensemble multiparticle sizing.

\section{IMAGING}

Imaging techniques are the most accurate and reliable methods and are well established and relatively inexpensive. Microphotography with focus discrimination has been used for many years for global characterization of particle laden flows and for detailed particle size and shape analysis. The number of particles that can be examined is limited by the field of view, the depth of field, the frequency of taking photographs, and the tedium of direct measurement of size and shape of individual particles. Holography provides "frozen" images of particles in three-dimensional sections of the flow field. After reconstruction of the hologram, sizing and shape analyses are made at various planes along the line of sight, during which focus discrimination is required. Cinematography and video recording provide motion pictures from which dynamic changes can be readily visualized and demonstrated, but sizing requires frame-by-frame analysis. The use of multiple flash lights and multiple pulsed lasers yields information on particle tracks and trajectories from which particle velocity and angle of flight can be determined. Changes in particle size as a result of vaporization, devolatilization, swelling, and chemical reaction can be recorded and measured.

Automatic digital image analysis techniques have developed rapidly as substantial funding has been made available for research and development of pattern recognition systems for analyzing visual information provided by satellites, missiles, and high speed aircraft in the fields of space, defense, aeronautics, aviation, and meteorology. This development is the result of requirements for 
identifying, monitoring, and analyzing images of objects such as tanks, ships, aircraft, missiles, clouds, and objects in outer space. Image enhancement is used to improve signal-to-noise ratios. Patterns are recognized, identified, categorized, and subsequently analyzed for size, shape, and location in space. Focus discrimination is made by measurement of intensity variation. Analog signals recorded by still, motion picture, and video cameras are transformed to digital recordings, and, where possible, direct recording is made with digital diode array cameras. Computers and software have been developed specially for automatic digital image analysis. These developments, coupled with ongoing research in three-dimensional photography, cinematography, and photogrammetry, are leading to a revitalization of imaging techniques that will result in more widespread usage and application of imaging for particle size and velocity analysis.

\section{SINGLE PARTICLE COUNTERS}

Laser anemometry using dual beams for Doppler frequency detection of scattered light signals from particles crossing interference fringes in the crossed beam measurement volume is now a well-established diagnostic technique for particle and gas (by particle seeding) velocity measurement. Using argon-ion and helium-neon lasers, beam splitters, lenses, photomultipliers, signal processors, digital clocks, and microprocessors, complete commercial packages are available. Measurements of velocity covering ranges from millimeters per second in creeping flows to thousands of meters per second in supersonic and hypersonic flows have been made in a variety of systems, including such complicated systems as blood flow, rotating helicopter blade wakes, hypersonic wind tunnels, aircraft in flight, and large industrial furnaces and internal combustion engines covering high temperature, high altitude, high speed, and high particle number density vaporizing and reacting flow systems. With extreme care and precision, frequency responses of $100 \mathrm{kHz}$ are attainable.

It has long been recognized that the amplitude of Doppler signals from laser Doppler velocimeter (LDV) systems is directly related to particle size. Simultaneous measurement of size and velocity of single particles is possible from measurements of amplitude, signature, and frequency of single Doppler signals. Several investigators have struggled for more than a decade to overcome the practical difficulties of achieving this objective. The Gaussian distribution of intensity across laser beams leads to a nonuniform intensity within measurement volumes so that the amplitude of signals is both a function of size and location within the measurement volume; large particles crossing the edge of the measurement volume can generate the same peak amplitude as small particles crossing the center of the volume. This problem of spatial ambiguity has been addressed, but not fully solved, by a number of different approaches. Use of ratios such as maximum (peak) and minimum (pedestal) values for visibility was recommended many years ago, but despite many claims, substantial and concrete evidence is available to show, both theoretically and experimentally, that visibility does not provide a unique or viable means for accurate particle sizing. Another approach to the spatial ambiguity problem is to restrict measurement to the central portion of the volume where intensity is constant by using off-axis collection or using $90^{\circ}$ detection for gathering of signals from particles that pass through the central region. Expansion of laser beams and "clipping of the Gaussian wings" can, in principle, yield uniform tophat distributions that remove the spatial ambiguity; this method has been tried but is not fully proven. Yet another approach has been to use statistical analyses based on the probability of particles crossing different locations within the volume; intensity deconvolution is used to solve (mathematically) the unknown scattering intensity and cross-section weighting to make statistical error estimates. The intensity deconvolution method does not allow the direct and simultaneous measurement of particle size and velocity of individual particles. Reliance is placed on statistical techniques. Mathematical techniques are complex, and software has not been fully developed. The system has not, as yet, been commercialized.

All of the single particle counters currently in use for particle sizing have problems associated with either alignment, spatial ambiguity, multiple scattering, inversion techniques, software, and/or data analysis so that expert developers and users have yet to agree as to which is the most viable solution. It is also important to recognize that each one of the approaches and systems has limitations and complexities that have, so far, prevented the development of a single commercial instrument that overcomes all the problems. Much additional research, development, and testing of techniques are needed, and calibration methods are required before these instruments can be recommended for usage by nonexperts.

The phase/Doppler analyzer instrument based upon the relative phase shift of the scattered light detected by several photomultipliers is a novel approach that is based on sound theoretical arguments and has the possibility of overcoming many of the alignment and interference problems that are associated with single particle counters. One prototype has been built and tested, indicating the feasibility and relative simplicity of this instrument, but clearly much more time and usage are required before the phase/Doppler spray analyzer could be proven superior to other single particle counters.

\section{ENSEMBLE MULTIPARTICLE SIZING}

For rapid, accurate, and simple analysis of the size distribution of clouds of particles, the commercially available laser diffraction particle size analyzers are the most viable, least expensive, and simplest to use, and are an effective means of making global assessments of the average size of particles. The instruments have been commercially available for several years and are in wide usage. Extensive experience has been obtained and presented in papers, reports, and discussions.

Laser diffraction techniques observe aggregate scattering properties, use inversion to reconstruct size distribution, can be used for line-of-sight or local measurements, and utilize less complex optical, electronic, and computing components than most other particle sizing instruments do. No velocity information is available on individual particles. Forward scattering based on Fraunhofer diffraction is utilized for measurement of scattered light intensity distributions recorded on annular photodiode array detectors.

Monolithic photodiode detectors currently can only be manufactured with sensitivity accuracy to within $20 \%$. The relative sensitivity differences between detector elements affect the measured diffraction signature. By a relatively simple calibration of the individual detectors in each particular instrument, this source of error can be eliminated. Monodisperse droplet generators, glass microspheres, standardized spray nozzles, and spray holograms have been used for calibration of individual instruments. Photomask calibration reticles consisting of an array of thin chrome film circles on a transparent glass substrate are commercially available. These photomasks simulate Rosin-Rammler distributions of spherical particles and can be very simply and effectively used to calibrate laser diffraction instruments. Limitations of particular lenses can be easily tested by placing the photomask at various locations and angles in the laser beam, yielding calibration factors that can be inserted in the computer programs. The calibration of the individual detectors and repeated calibration with photomasks make very significant improvements in the accuracy and reliability of individual instruments.

\section{SUMMARY OF PAPERS IN THIS SPECIAL ISSUE}

Although great strides have been taken in obtaining particle size and trajectory measurements in flowing streams, much work still remains to be done. The papers in this issue represent current areas of research addressing problems in imaging, single particle 
counting, and ensemble multiparticle sizing. In addition, most particle measurement devices are developed and characterized in well-controlled laboratory experiments. Therefore, it is important to realize the behavioral differences experienced under actual experimental conditions. In the opening paper, J. Tishkoff addresses the practical problems of extending devices used in well-controlled laboratory experiments to measurements on "real" systems. He particularly expresses concern for the development of standard operating procedures and the development of standard reference materials for calibration, both of which are needed to establish credibility and reliance for nonideal testing conditions.

The next three papers address the development of automated systems for image data analysis. In the first paper, B. Weiss et al. describe a direct imaging method in which images of silhouetted droplets are projected onto a vidicon tube. A microprocessor can provide droplet size histograms automatically and in real time over a particle range of 6 to $1200 \mu \mathrm{m}$. For non-in-situ measurements, P. Malyak and B. Thompson, and A. Stanton et al. describe automated techniques for analyzing holographic images of the flow field. The use of holograms allows the determination of particle size and also provides information on particle velocity and trajectory. Malyak and Thompson use the optical Fourier transform plane of a doubly exposed hologram to measure particle displacement and velocities. In this system, the fringe frequency and orientation are related to the magnitude and direction of a particle's displacement or velocity. Stanton et al. also report on the automated image analysis of holograms; however, they use analysis patterns in two or more nonimage planes to determine particle size and location. This approach provides both speed and accuracy while excluding hologram artifacts.

The use of light scattering techniques for single particle counting is the subject of the next three papers. W. Bachalo and $M$. Houser present a method that uses the interference fringe pattern produced by scattered light to obtain size information. They report a significant advantage resulting from a lack of sensitivity to scattered light and fringe visibility, as well as a lack of susceptibility to practical effects such as optical imperfection, environmental conditions, and alignment. D. Holve and K. Annen review the field of single particle counting and describe in detail the use of intensity deconvolution for optical particle counting, sizing, and velocimetry. They present results obtained from in situ measurements on laboratory and industrial facilities using both forward scatter and backscatter geometries. C. Hess and V. Espinosa discuss theoretical aspects and results of a nonintrusive technique using either lasers of two colors or two polarizations of the same color laser to identify a probe volume of known illumination for measuring absolute scattered light intensity. This configuration provides a self-calibrating technique with a dynamic range in excess of 30 from which particle size and velocities can be measured.

The final four papers of the issue discuss ensemble multiparticle sizing. E. Hirleman et al. evaluate in detail the effects of optical sample volume and nonideal lenses on the response characteristics for a Malvern 2200 laser diffraction particle size analyzer. As a result of this study, a set of calibration factors was derived to correct for the nonideal response. The effect of particle position was also evaluated experimentally and compared to model predictions. B. Ewan et al. report on the use of the Malvern ST1800 droplet sizing instrument to analyze holographic records of a transient spray. Although this is a unique approach to automated image analysis, emphasis of the paper is on droplet size analysis and droplet behavior in complicated sprays. In his paper L. Dodge also addresses the practical problems associated with calibration procedures for diffraction-based particle sizing instruments. An empirical scheme is developed to compensate for changes in calibration resulting from multiscattering in dense sprays. In the final paper, G. Gouesbet and M. Ledoux review their experience in particle sizing using various optical techniques at Rouen University. In this review, they cover a broad range of particle size measurement devices. Techniques specifically considered include corrected (top-hat) laser beam methods, Gabor holography, Malvern diffractometry, and diffusion broadening spectroscopy. 